UNIMAS E-Journal of Civil Engineering, Vol. 3 (Special Issue) 2012

\title{
Road Pavement Stiffness Determination using SASW Method
}

\author{
M. A. Ismail, A. R. Samsudin, A. G. Rafek and K. A. M. Nayan
}

\begin{abstract}
The Spectral Analysis of Surface Waves (SASW) method is an in-situ seismic technique for the evaluation and assessment of road pavement. The method is based on the theory of stress waves propagating in elastic media with the key elements being the generation and detection of Rayleigh wave motion. A set of transient impact source with a range of frequencies is used to generate the Rayleigh wave energy that is able to propagate along the surface layer of the pavement. Through two vertical accelerometers, the motion of the wave for each range of frequency is recorded and calculated using a dynamic signal analyzer. A dispersion curve is then constructed from the cross-power spectrum of the Fast Fourier Transform (FFT) that results in the phase velocity versus wavelength plot. An iterative inversion is then carried out to obtain the shear wave velocity profile with depth and the corresponding dynamic modulus of each pavement units. This paper presents a case study carried out on a new road pavement construction site. It was found that the profile of the dynamic shear and Young's modulus versus depth obtained from SASW method was able to detect each layer of the pavement units. This finding would be useful in the assessment of the pavement integrity.
\end{abstract}

Keywords: NDT, pavement, Rayleigh wave, surface wave, dynamic modulus

\section{INTRODUCTION}

$\mathrm{T}$ he Spectral Analysis of Surface Waves (SASW) method is an in situ non-destructive testing technique for the evaluation of the dynamic modulus and thickness of layered media. This method is based on the dispersive nature of Rayleigh wave in order to obtain the shear wave velocity profile for the determination of the material dynamic properties.

The purpose of this study is to use the SASW method to determine the dynamic modulus and thickness of flexible pavement layers, although it can be equally applied to rigid pavements. The field procedure, data analysis technique and the interpretation of this method are described in this paper. The case study of a newly constructed road in Putrajaya is also presented to demonstrate its potential and ability in the pavement evaluation.

M.A. Ismail is with the Industrial Technology Division, Malaysian Nuclear Agency, Bangi, 43000 Kajang, Selangor, Malaysia; email: azmi@nuclearmalaysia.gov.my

A.R. Samsudin and A.G. Rafek are with the Department of Geology, Faculty of Science and Technology, Universiti Kebangsaan Malaysia, 43600 Bangi, Selangor, Malaysia; email: abrahim@pkrisc.cc.ukm.my; aghani@pkrisc.cc.ukm.my

K.A.M. Nayan is with the Department of Civil \& Structural Engineering, Faculty of Engineering, Universiti Kebangsaan Malaysia, 43600 Bangi, Selangor, Malaysia;email: khairul@ vlsi.eng.ukm.my 


\section{METHODOLOGY}

\section{A. Propagation of Rayleigh Waves}

The SASW method is based on the particles motion of Rayleigh wave in heterogeneous media. The energy of Rayleigh waves propagates mechanically along the surface of a media and their amplitude decrease rapidly with depth. Particle motions associated with Rayleigh wave are composed of both vertical and horizontal components, which when combined, form a retrogressive ellipse close to the surface, as shown in Figure 1(a).

In homogenous, isotropic, elastic half-space, Rayleigh wave velocity does not vary with frequency. However, Rayleigh wave velocity varies with frequency in the layered medium where there is a variation of stiffness with depth. This phenomenon is termed dispersion where Rayleigh wave refers to the variation of phase velocity as a function of wavelength. The ability for detecting and evaluating of the depth of the medium is influenced by the wavelength and the frequency generated. Figure 1(b) shows that the shorter wavelength of higher frequency penetrates the shallow zone of the near surface and the longer wavelength of the lower frequency penetrates deeper into the medium.

\section{B. Dynamic Properties Materials}

Rayleigh wave propagation technique may be used to determine the parameters of the dynamic modulus of in situ pavement and soil layers. Values of these parameters determined represent the material behaviour at small shearing strain, of less than of $0.001 \%$ [15]. In this strain range, material behaviour, particularly sub grade material, is typically independent of strain amplitude. Nazarian and Stokoe [12] have explained that the modulus parameter of material is constant below a strain of about $0.001 \%$ and is equal to $E_{\max }$ and its corresponding $G_{\max }$.

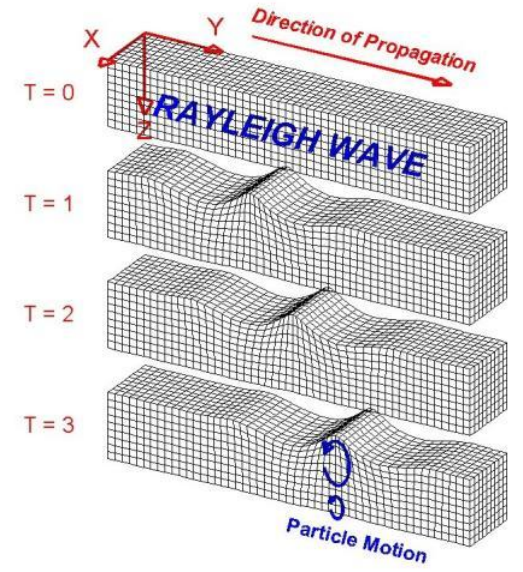

(a)

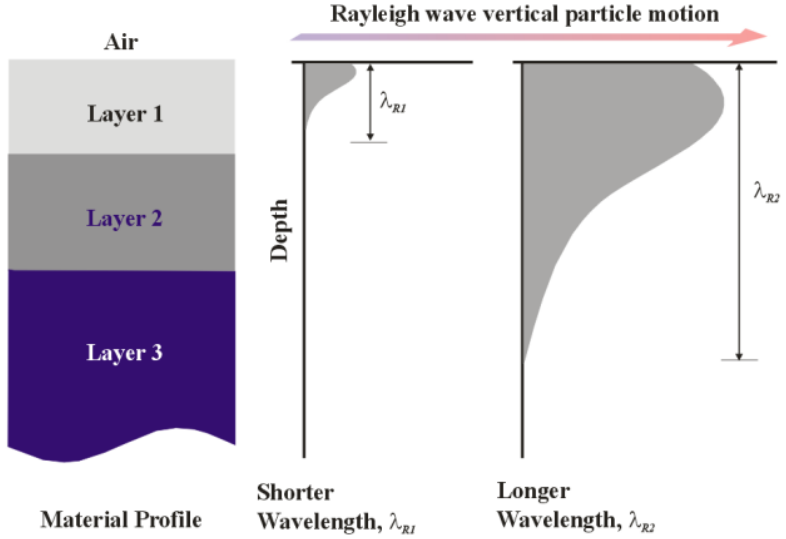

(b)

Figure 1 (a) The particle motion and direction of Rayleigh propagation in a homogeneous medium, and (b) Variation of vertical particle motion for Rayleigh waves with different wavelengths

The moduli of the pavement materials can be determined from the following equations [18]:

$$
\begin{aligned}
& G=\frac{\gamma}{g} V_{s} \\
& E=\frac{2 \gamma}{g} V_{s}^{2}(1+\mu) \\
& E=2(1+\mu) G
\end{aligned}
$$


where, $G=$ dynamic shear modulus, $E=$ dynamic modulus of elasticity, $V_{s}=$ shear wave velocity, $\gamma=$ total unit weight of the pavement or soil, $g=$ gravitational acceleration and $\mu=$ Poisson's ratio.

\section{The Development of SASW Technique}

The first experiment in layered media involving Rayleigh wave was developed by Jones [7] and is known as the steady state method. This method was time consuming and had produced high inaccuracies of the results. Recently, the development of more powerful computers for signal processing and for numerical analysis has enabled the SASW method to be more practical. Latter, the SASW method was improved with the generation of the dispersion curve and the affected factors in the calculation of the elastic modulus and the thickness of the pavement and the soil profile being studied [12].

Over the past decade, the SASW method has been utilised in different applications. These applications include characterisation of soils for foundation [10], evaluation of concrete structures [2], detection of anomalies [4], detection of the structural layer of cement mortar [2], compaction assessment of filled structures [9] and the evaluation of the structural integrity of the railway ballast [19].

\section{Measurement Procedures}

The test is performed using a vertical impact source applied on the surface layer of pavement profile and detecting the transient signals by two receivers (geophones and accelerometers) placed along a straight line starting from the impulse point. The signals are recorded using a dynamic spectrum analyser for post processing as shown in Figure 2(a). Figure 2(b) shows one of the test sites.

Changing receivers spacing and using different sources which are characterized by the frequency range of the impulse are required to sample different depths. Short receiver spacing with high frequencies (short wavelength) are used to sample shallow layers while long receiver spacing with low frequencies (long wavelengths) are used in sampling deeper layers.

A Fast Fourier Transform in the spectrum analyser transforms the recorded signals in the time domain from the receivers to frequency domain. The coherence between the signals and the phase information of the transfer function are evaluated from the output of the spectrum analyser and are saved for further processing, as shown in Figure 3 (a). The transfer function spectrum is used to obtain the relative phase shift between the two signals in the range of the frequencies being generated, as shown in Figure 3(b).

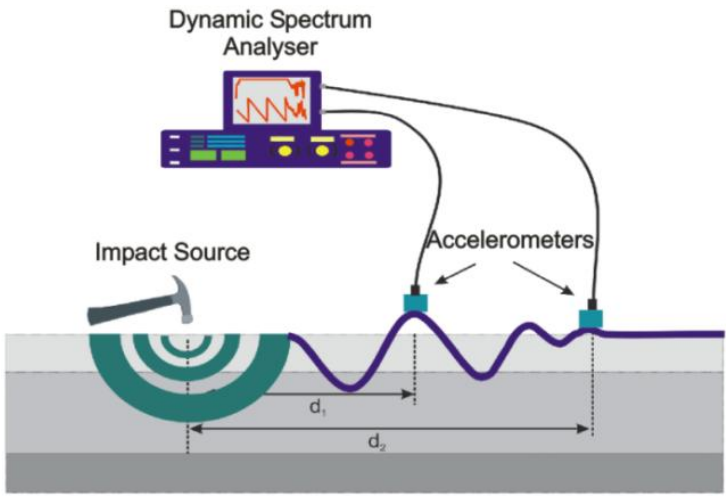

(a)

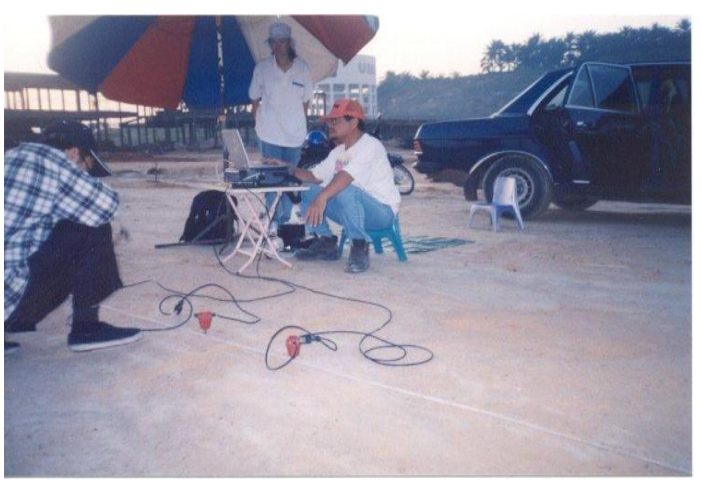

(b)

Figure 2 (a) The set up for the SASW measurement and (b) The test site 


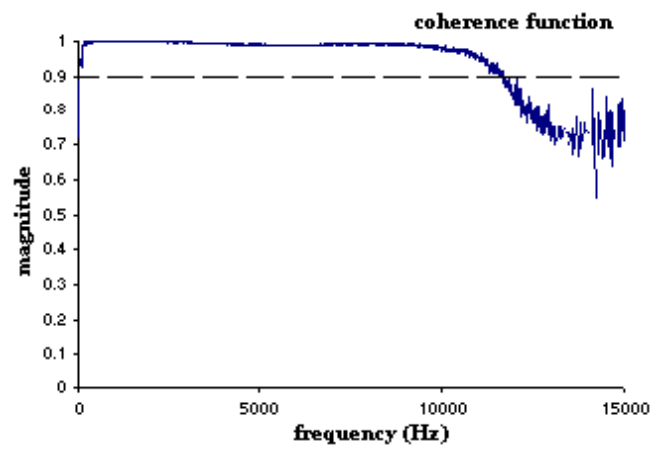

(a)

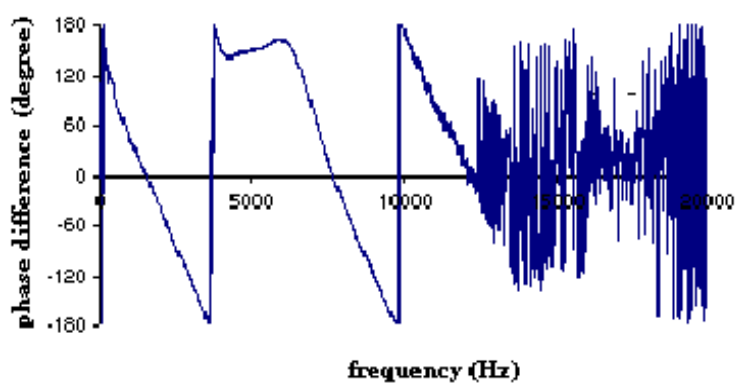

(b)

Figure 3 (a) The coherence function, and (b) A typical phase plot of the transfer function on an asphalt concrete

\section{E. Development of Dispersion Curve}

Based on the dispersion characteristics of Rayleigh waves in layered media, a plot of wavelength versus phase velocity is known as the dispersion curve. For a travel time equal to the period of the wave, the phase different is $360^{\circ}$. Thus, for each frequency the travel time between receivers can be calculated by

$$
t(f)=\frac{\phi(f)}{360 f}
$$

where $f$ is the frequency, $t(f)$ is the travel time and $\phi(f)$ is the phase difference in degrees at a given frequency. The distance of the receiver $(d)$ is a known parameter. Therefore, Rayleigh wave velocity at a given frequency $\left(V_{R}(f)\right)$ is simply calculated by:

$$
V_{r}(f)=\frac{d}{t(f)}
$$

The corresponding wavelength of the Rayleigh wave, $L_{R}(f)$ is equal to:

$$
L_{r}(f)=\frac{V_{r}(f)}{f}
$$

By repeating the procedure outlined by Equation (1) through (3) for every frequency, the Rayleigh wave velocity corresponding to each wavelength is evaluated and the experimental dispersion curve is generated. It is important to note that velocities obtained from the experimental dispersion curve are not actual Rayleigh wave velocities, but rather apparent or phase velocities. The existence of a layer with a higher or lower velocity at the surface of a medium affects the measurement of the velocities for the underlying layers. Thus, a method for evaluating actual Rayleigh wave velocities from apparent Rayleigh wave velocities is necessary in SASW test. 


\section{F. Inversion of Dispersion Curve}

The process of determining the actual propagation velocities at different depth (velocity profile) from the experimental dispersion curve is known as the inversion of the Rayleigh wave dispersion curve. The propagation theory models a theoretical dispersion curve, which is compared with experimental dispersion curve.

Joh [6] has suggested two methodologies for the evaluation of a stiffness profile: the iterative forward modeling analysis and the inversion analysis. The iterative forward modeling analysis requires an initial guess (priori) of the stiffness profile. To each layer a thickness, a shear wave velocity, a Poisson's ratio (or compression wave velocity), and a mass density are assigned. Many studies have been carried on about the influence of each one of these parameters and the general conclusion is that the influence of density and Poisson ratio is negligible [11]. Therefore, they can be estimated on the basis of experience without any sensible effects on the final result. Based on these initial priori parameters of the initial profile, a theoretical dispersion curve is calculated using stress wave propagation theory. The theoretical dispersion curve is compared with the experimental dispersion curve. If the two dispersion curves do not match, the initial profile (number of layers, layer thickness, shear wave velocity, or any combination) is adjusted, and another theoretical dispersion curve is calculated. The trial-and-error procedure is repeated until the two curves match, and then the associated assumed profile is considered the real profile.

\section{CASE STUDIES}

Case Study 1: Landed-Zone 4-B, Precint 11, Putrajaya

The SASW testing was carried out on a council road pavement surface at Landed-Zone 4-B, Precint 11, Putrajaya construction project (PREC11-JP1 line). The pavement profile consists of an asphalt layer (100 mm thick), a base of crushed aggregates (300 mm thick), and a sub base of sand (200 mm thick) over a meta-sediment sub grade. Material properties and thickness of the four layers are shown in Figure 4(a). In this study, the receiver spacing of $10,20,40,80,160$ and $320 \mathrm{~cm}$ were used.

The result of the inversion is as shown in Figure 4(b). A total of 10 layers were used in this inversion analysis and the thickness of the layers was increased gradually as the properties of the near surface material are of most interest in a pavement system. By assuming reasonable values of Poisson's ratio and mass density of each layers, the dynamic Young's modulus profile is calculated using Eq. (2) and (3) and is plotted in Figure 4(c). The modulus and depths determined by the SASW measurements from the inversion are in good agreement with each layer of the pavement profile. A zone with higher modulus is interpreted from 0 to $10 \mathrm{~cm}$ for the asphalt concrete layer that has the highest stiffness properties $(100-1000 \mathrm{MPa})$. An abrupt decrease of the modulus then occurs at the transition layer of the base followed by a gradual increase of the modulus for the sub base and sub grade with elastic modulus of around $10 \mathrm{MPa}$.

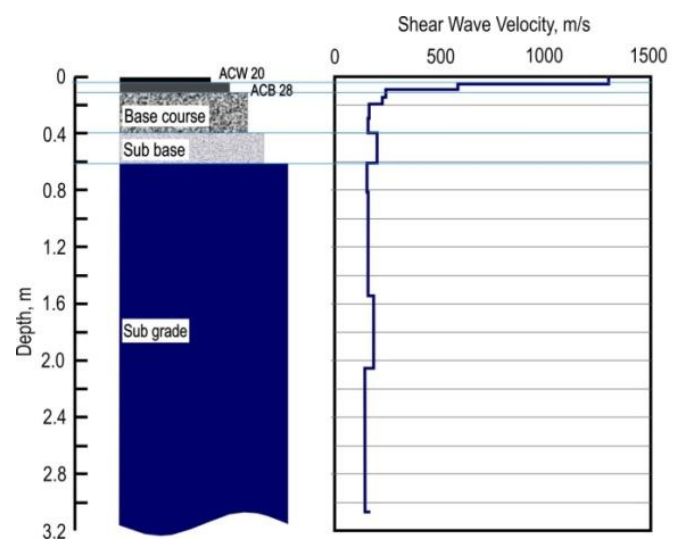

(a) (b)

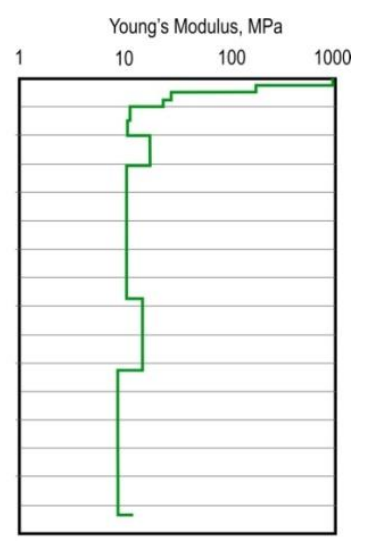

(c)

Figure 4 (a) The designed pavement profile, (b) the final shear wave profile, and (c) the calculated dynamic Young modulus profile for PREC11-JP1 line 
Case Study 2: Km 296, North - South Expressway

The SASW testing was carried out on a pavement surface at Km 296 of the North-South Expressway during rehabilitation works (LRSB-ApJ line). The pavement structure consists of an asphalt layer (180 - $200 \mathrm{~mm}$ thick), a sub base of crushed aggregates and fine sand (2000 - $2500 \mathrm{~mm}$ thick), over a metasediment sub grade. Material properties and thickness of the four layers are shown in Figure 5(a). In this study, the receiver spacing of 10, 25, 50, 100 , and $200 \mathrm{~cm}$ were used. The sources for the Rayleigh wave employed were slightly different from those in the case study 1 . For high frequency signals, 2-inch nail and small hammers were used, geological hammer was used for receiver spacing higher than $100 \mathrm{~cm}$.

The shear wave velocity profile along with the layering use in this inversion is shown in Figure 5(b). RMS data error obtained for this inversion analysis is $20.75 \%$. The dynamic Young's modulus profile is calculated and is plotted in Figure 5(c). The profiles determined by the SASW measurements show good agreement with the pavement profile. A zone with higher modulus is interpreted from 0 to $20 \mathrm{~cm}$ for the asphalt concrete surface layer that has the highest stiffness properties $(100-1500 \mathrm{MPa})$.

\section{Concluding Remarks}

The thickness of layering obtained from the inversion analysis compared well with the existing profile obtained from the construction record. This surface layer of LRSB-ApJ line line is slightly stiffer than the PREC11-JP1 line as a more stringent specification was applied to the former. The shear wave velocities and dynamic Young's modulus profiles from this study were compared with SASW testing that had been carried out by other workers, such as Nazarian (1984) and Al Hunaidi (1998) (Table 1). In general, there is a reasonable agreement between the results from these studies.

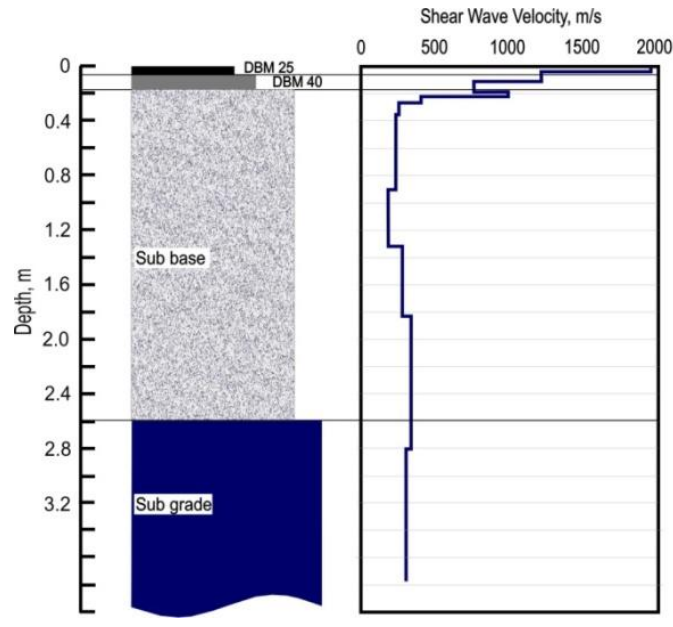

(a) (b)

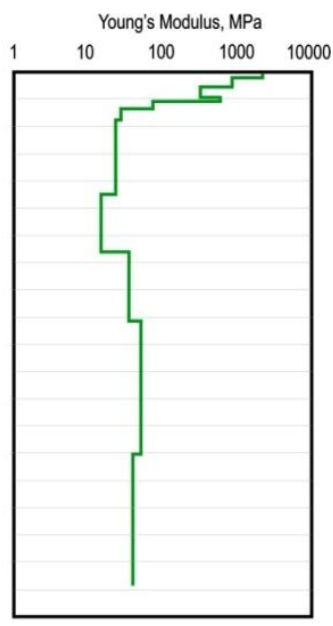

(c)

Figure 5 (a) The pavement structure profile, (b) The final shear wave profile, and (c) the calculated dynamic Young modulus profile for LRSB-ApJ line. 
UNIMAS E-Journal of Civil Engineering, Vol. 3 (Special Issue) 2012

Table 1 The frequency range produce by the ball bearings and the impact hammers

\begin{tabular}{|c|c|c|c|c|c|}
\hline \multirow{2}{*}{ Steel Ball Bearing (g) } & \multicolumn{2}{|c|}{ Freq. Range (kHz) } & \multirow{2}{*}{ Impact } & \multicolumn{2}{|c|}{ Freq. Range (Hz) } \\
\cline { 2 - 3 } \cline { 5 - 5 } & Lowest & Highest & Hammer (kg) & Lowest & Highest \\
\hline 5 & 8 & 20 & 0.19 & 962 & 2575 \\
15 & 8 & 18 & 0.58 & 556 & 1719 \\
30 & 4 & 12 & 1.1 & 488 & 1575 \\
45 & 2 & 8 & 1.92 & 406 & 1088 \\
65 & 1 & 3 & 4.87 & 337 & 944 \\
\hline
\end{tabular}

The shear wave velocities profile from this study were also compared to results from the SASW testing that had been carried out by Al Hunaidi [1] and Nazarian [11] is described in Table 2. In general, there is a reasonable agreement between the results from this study and their results. The data obtained from Nazarian [11] were from the test on concrete pavement while the data obtained from Al Hunaidi [1] were summarized for both the asphalt and concrete pavements.

Table 2 Comparison of shear wave velocity from this study to that of Nazarian [11] and Al-Hunaidi [1]

\begin{tabular}{|c|c|c|c|}
\hline \multirow{2}{*}{ Profile } & \multicolumn{3}{|c|}{ Shear wave velocity $(\mathbf{m} / \mathbf{s})$} \\
\cline { 2 - 4 } & This study & Nazarian [11] & Al Hunaidi [1] \\
\hline Surface course & $900-1100$ & 2425 & $1000-2500$ \\
Base course & $150-200$ & 176.9 & $100-500$ \\
Sub base course & $195-270$ & 301.95 & $100-500$ \\
Sub grade & $200-350$ & 271.45 & $80-300$ \\
\hline
\end{tabular}

\section{CONCLUSIONS}

The SASW method has managed to characterize all the layers of the pavement profile in terms of shear wave velocity and dynamic shear and elastic modulus satisfactory. The method can be useful to be applied in the early stages of pavement evaluation for detecting the dynamic properties and thicknesses of the profile. This method has been also found to be able to detect sandwiched low modulus layers reasonably accurate, unlike the drawbacks from the other seismic methods for this case.

The SASW method has managed to characterize all the layers of the pavement profile in terms of shear wave velocity and dynamic shear and elastic modulus satisfactory. The method can be useful to be applied in the early stages of pavement evaluation for detecting the dynamic properties and thicknesses of the profile. This method has been also found to be able to detect sandwiched low modulus layers reasonably accurate, unlike the drawbacks from the other seismic methods for this case.

\section{ACKNOWLEDGEMENTS}

We would like to give our sincere appreciations to Miss Raja Azizah binti Raja Yeop (Perembajaya Engineering Sdn. Bhd.) for the permission and to the data of the site. We would also like to thank Prof. Dr. Sung Ho Joh (ChungAng University) for his input during the data processing work.

\section{REFERENCES}

[1] Al-Hunaidi, M.O., 1998. Evaluation-based genetic algorithms for analysis of non-destructive surface waves test on pavements, $N D T \& E$ international, Vol.31, No.4, pp.273-280.

[2] Cho, Y.S. and Lin, F-B, 2001. Spectral analysis of surface wave response of multi-layer thin cement mortar slab structure with finite element thickness, ND\&T E International 34 (2001), Elsevier Science, pp.115-122. 
UNIMAS E-Journal of Civil Engineering, Vol. 3 (Special Issue) 2012

[3] Cho, Y.S., 2002, NDT response of spectral analysis of surface wave method to multi-layer thin high strength concrete structure, Ultrasonic, 2336, Elsevier Sciences.

[4] Gucunski, N., 2000. Field Implementation of Surface Waves for Obstacle Detection (SWOD) Method. $15^{\text {th }}$ WCNDT, Roma 2000

[5] Hossain, M.M., and Drnevich, V.P., 1989. Numerical and optimisation techniques applied to surface waves for back-calculation of layer moduli. In Non-destructive testing of pavements dan back-calculation of moduli. Edited by Bush, A.J., III, dan Baladi, G.Y. American Society for Testing dan Materials, Special Technical Publication 1026, pp. 649-669.

[6] Joh, S.-H., 1996. Advances in data interpretation technique for Spectral Analysis-of-Surface-Waves (SASW) measurements. Ph.D. Dissertation, the University of Texas at Austin, Austin, Texas, U.S.A., 240 pp.

[7] Jones, R.B. 1958. In-situ measurement of the dynamic properties of soil by vibration methods. Geotechnique. Vol.8.(1).pp.1-21.

[8] Kausel, E., and Röesset, J.M., 1981. Stiffness matrices for layered soils. Bull. Seismol. Soc. Am., 72, pp 1743-1761.

[9] Kim, D.S, Shin M.K and Park H.C., 2001. Evaluation of density in layer compaction using SASW method. Soil Dynamic and Earthquake Engineering 21 (2001), Elsevier Science, pp.39-46.

[10] Madshus, C., and Westerdahl, H., 1990. Surface wave measurements for construction control and maintenance planning of roads and airfields. Proc. $3^{\text {rd }}$. Int. Conf. On Bearing Capacity of Roads and Airfields, July 3-5, Trondheim, Norway.

[11] Nazarian, S., 1984. In-situ determination of elastic moduli of soil deposits and pavement systems by Spectral-Analysis-Of-Surface-Wave Method. Ph.D. Dissertation, University of Texas at Austin, $452 \mathrm{pp}$.

[12] Nazarian, S. and Stokoe II, K. H. 1984. In-situ shear wave velocity from spectral analysis of surface waves. Proc. $8^{\text {th }}$ World Conf. On Earthquake Engineering, 3, pp 31-38.

[13] Rix, G.J., Bay, J.A, and Stokoe II, K.H., 1990. Assessing in situ stiffness of curing Portland cement concrete with seismic tests. Paper presented to Annual Meeting, Transportation Research Board, Washington, D.C., January.

[14] Stokoe II, K.H., Wright, S.G., Bay, J.A, and Roesset, J.M., 1994. Characterization of geotechnical sites by SASW method. Geotechnical characterization of sites, R.D. Wood, ed., Oxford and IBH Publishing Co., New Delhi, India, 15-26.

[15] Stokoe II, K.H. and Hoar, J.H.. 1978. Variable affecting in situ seismic measurement. Proc. Conference on Earthquake Engineering and Soil Dynamic, ASCE, Pasadena, CA., Vol.II,pp.919-939.

[16] Thomson, W.T. 1950. Transmission of elastic waves through a stratified solid medium. Jour. of Appl. Phys., Vol. 21, Feb. 1950, pp 8993.

[17] William, O. 1981. Rayleigh wave velocity measurement using broad band frequency sources, Miscellaneous Paper EL-81-3, US Army Engineer Waterways Experiment Station, Vicksburg.

[18] Yoder, E.J. and Witczak, M.W, 1975. Principle of pavement design. John Willey \& Sons, New York.

[19] Zagyapan, M. and Fairfield, C.A., 2002. Continuous surface wave and impact methods of measuring the stiffness and density of railway ballast, NDT\&E International 35 (2002), Elsevier Science, pp.75-81. 